\title{
CRESCIMENTO DE Dalbergia nigra (Vell.) Allemão ex Benth SOB DIFERENTES DOSES DE CÁLCIO, MAGNÉSIO E ENXOFRE ${ }^{1}$
}

Elzimar de Oliveira Gonçalves², Haroldo Nogueira de Paiva ${ }^{3}$, Julio Cesar Lima Neves ${ }^{4}$ Valéria Hollunder Klippel $^{5}$ e Marcos Vinicius Winckler Caldeira²

\begin{abstract}
RESUMO - O jacarandá (Dalbergia nigra (Vell.) Allemão ex Benth) é uma espécie da família LeguminosaeFabaceae, com potencial para diversos usos, incluindo recuperação de áreas degradadas. Neste trabalho, objetivou-se verificar o crescimento das mudas de jacarandá-da-bahia sob diferentes doses dos nutrientes cálcio, magnésio e enxofre, em solos com características diferentes. O experimento foi conduzido em casa de vegetação no período de fevereiro de 2006 a junho de 2006. Os tratamentos foram delimitados segundo uma matriz baconiana, em que se variaram os nutrientes em três doses e dois tratamentos adicionais (zero e referência). Adotou-se o delineamento inteiramente casualizado com quatro repetições, num total de 44 unidades amostrais. As mudas foram conduzidas em vasos plásticos com capacidade para 2,1 dm $\mathrm{dm}^{-3} \mathrm{e}$ colhidas aos 120 dias após a semeadura, sendo avaliadas diversas características morfológicas. A espécie se mostrou, em geral, pouco exigente em relação à aplicação dos nutrientes. Enquanto novos estudos, com doses menores às testadas neste, não sejam feitos, e tomando como base a massa seca total e as condições similares à desse experimento, indicam-se as doses de: $0,8 \mathrm{cmol}_{\mathrm{c}} \mathrm{dm}^{-3}$ de solo de Ca e $20 \mathrm{mg} \mathrm{dm}^{-3}$ de solo de $\mathrm{S}$, independente do solo testado; e 0,2 e 0,8 $\mathrm{cmol}_{\mathrm{c}} \mathrm{dm}^{-3}$ de solo de Mg para solos com características similares ao PVAm e LVAd, respectivamente
\end{abstract}

Palavras-chave: Nutrição mineral; Espécies florestais nativas; Jacarandá-da-bahia.

\section{DEVELOPMENT OF Dalbergia nigra (Vell.) Allemão ex Benth UNDER DIFFERENT DOSES OF CALCIUM, MAGNESIUM AND SULPHUR}

\begin{abstract}
The jacaranda (Dalbergia nigra (Vell.) Allemão ex Benth) is a specie with potential for several uses, including recovery degraded areas. The objective of this work was to evaluate the effect of calcium, magnesium and sulfur, on the seedlings growth under different soil types. The experiment was conducted in a greenhouse from February 2006 to June 2006. The treatments were delimited according to a Baconian matrix where nutrients are varied in three different doses and two additional treatments (zero and reference). A complete randomized design with four replications was established in a total of 44 sample units. The seedlings were grown in pots with a capacity for $2.1 \mathrm{dm}^{-3}$, and collected at 120 days after sowing and evaluated several morphological characteristics. The specie was generally undemanding regarding the application of nutrients, and the doses recommended when classified as low by the hand of fertilization. While further studies with doses lower than those tested in this, are not made, and building on the total dry mass and conditions similar to this experiment, are indicated doses: $0.8 \mathrm{cmolc} \mathrm{dm}^{-3}$ of soil Ca and $20 \mathrm{mg} \mathrm{dm}^{-3}$ of soil S, regardless of the test soil; and 0.2 and $0.8 \mathrm{cmolc} \mathrm{dm}^{-3}$ soil Mg to soils with similar characteristics to the PVAm and LVAd, respectively.
\end{abstract}

Keywords: Mineral nutrition; Native forest species; Jacaranda-da-bahia.

\footnotetext{
${ }^{1}$ Recebido em 28.04.2011 aceito para publicação em 27.02.2014.

${ }^{2}$ Departamento de Ciência Florestal e da Madeira, Universidade Federal do Espírito Santo. E-mail: <elzimar.goncalves@ufes.br> e<mvwcaldeira@gmail.com>.

${ }^{3}$ Departamento de Engenharia Florestal, Universidade Federal de Viçosa, UFV, Brasil. E-mail: <hnpaiva@ufv.br>.

${ }^{4}$ Departamento de Solos, Universidade Federal de Viçosa, UFV, Brasil. E-mail: <julio@solos.ufv.br>.

${ }^{5}$ Departamento de Engenharia Agrícola, Universidade Federal de Viçosa. E-mail: <valeria.h.klippel@hotmail.com>.
} 


\section{INTRODUÇÃO}

A melhoria da consciência ambiental por parte da população e a cobrança dos órgãos competentes sobre a aplicação da legislação florestal vigente têm contribuído para a crescente utilização de espécies florestais nativas no enriquecimento de matas e recuperação de áreas degradadas. Da mesma forma, o plantio dessas espécies para fins madeireiros ou para produção de frutos, cascas, resinas, óleos, entre outros, se constitui numa interessante alternativa, visando futuramente à oferta de madeira ou outros produtos diferentes dos obtidos pelos plantios com espécies exóticas. No entanto, explorar o potencial das espécies nativas ainda é difícil pela falta de estudos sobre vários aspectos do crescimento e desenvolvimento delas, entre eles as exigências nutricionais e a absorção de nutrientes na fase de muda.

Uma espécie de grande interesse para estudo é a Dalbergia nigra (Vell.)Allemão ex Benth, popularmente conhecida como jacarandá-da-bahia, pertencente à família Leguminosae-Fabaceae, encontrada nos Estados da Bahia, do Espírito Santo, de Minas Gerais, do Rio de Janeiro e de São Paulo, na Floresta Pluvial Atlântica (LORENZI, 2000). Sua madeira é mundialmente conhecida na construção de mobiliário de luxo e na fabricação de pianos. Utilizada também em acabamentos internos na construção civil, como lambris, molduras, portas, rodapés e em revestimento de móveis, caixas de rádios e televisão. A árvore é ainda muito ornamental, principalmente pela folhagem delicada e forma aberta de sua copa, tendo grande potencial para uso no paisagismo em áreas amplas (LORENZI, 2000). Ocorre naturalmente em solos de baixa fertilidade química, pouco exigente em $\mathrm{P}$, com preferência de solos com baixos teores de alumínio e pH superior a 5,2 (CARVALHO, 2003). É também uma espécie indicada na recuperação do solo, por depositar razoável camada de folhas e mostrar grande amplitude de tolerância ambiental.

Estudos sobre a produção de mudas de jacarandáda-bahia foram realizados por Reis et al. (1997), que, testando diferentes doses de $\mathrm{P}$, K e S em um Latossolo Vermelho-Amarelo álico, obtiveram resposta positiva à adição de $\mathrm{P}$ e $\mathrm{K}$ nas doses de $250 \mathrm{mg} \mathrm{dm}^{-3}$ e $30 \mathrm{mg}$ $\mathrm{dm}^{-3}$, respectivamente, e ausência de efeito da aplicação de $80 \mathrm{mg} \mathrm{dm}^{-3} \mathrm{~S}$. Bernardino et al. (2007) testaram o efeito da saturação por bases do substrato sobre o crescimento dessa espécie, concluindo que mudas de melhor qualidade são obtidas na saturação em torno de $60 \%$ quando cultivadas em um Argissolo. Marques et al. (2006), estudando o efeito de diferentes fontes de nitrogênio adicionados ao substrato Argissolo Vermelho-Amarelo, obtiveram maior massa seca total, tendo como fonte o sulfato de amônio na dose de 180 $\mathrm{mg} \mathrm{dm} \mathrm{m}^{-3}$ de $\mathrm{N}$.

O objetivo deste trabalho foi verificar o crescimento das mudas de jacarandá-da-bahia sob diferentes doses dos nutrientes cálcio, magnésio e enxofre, em solos com características diferentes.

\section{MATERIAL E MÉTODOS}

O experimento foi realizado no período de fevereiro a junho de 2006, em casa de vegetação, no Horto Florestal do Instituto Federal de Educação, Ciência e Tecnologia do Sudeste de Minas - Campus Rio Pomba, em Rio Pomba, MG. Situado na coordenada geográfica $21^{\circ} 14^{\prime}$ $31^{\prime \prime} \mathrm{S}$ e $43^{\circ} 09^{\prime} 39^{\prime \prime O}$ e altitude de $477 \mathrm{~m}$, apresentou temperatura média diária de $21,11^{\circ} \mathrm{C}$, máxima de 26,52 ${ }^{\circ} \mathrm{C}$ e mínima de $17,68{ }^{\circ} \mathrm{C}$.

Utilizaram-se como substrato na produção das mudas amostras de três classes de solos: Latossolo Vermelho-Amarelo distrófico (LVAd), Argissolo VermelhoAmarelo mesotrófico (PVAm) e Latossolo VermelhoAmarelo álico (LVAa) predominantes na região da Zona da Mata de Minas Gerais, segundo Resende et al. (2002). Essas amostras foram retiradas cerca de 0,30 m abaixo da camada superficial do solo, das quais se determinaram as características físicas e químicas (Tabela 1). As amostras, depois de serem secas ao ar livre, foram peneiradas em peneiras de malha de $5 \mathrm{~mm}$ e armazenadas em sacos plásticos e em local fechado, para uso em diversos experimentos.

Os tratamentos foram definidos a partir de uma matriz baconiana (TURRENT, 1979), consistindo de três doses de cada um dos nutrientes cálcio, magnésio e enxofre, além de um tratamento sem adição de nutrientes e outro com adição de todos os nutrientes, denominado como referência. O delineamento experimental foi inteiramente casualizado com quatro repetições.

As doses de nutrientes foram adicionadas nos substratos, mantendo-se os demais nutrientes iguais aos da referência, totalizando 11 tratamentos: 1- Referência ( $\mathrm{N}=100 \mathrm{mg} \mathrm{dm}^{-3}, \mathrm{P}=300 \mathrm{mg} \mathrm{dm}^{-3}, \mathrm{~K}=100 \mathrm{mg} \mathrm{dm}^{-3}$, $\mathrm{Ca}=1 \mathrm{cmol}_{\mathrm{c}} \mathrm{dm}^{-3}, \mathrm{Mg}=0,4 \mathrm{cmol}_{\mathrm{c}} \mathrm{dm}^{-3}, \mathrm{~S}=40 \mathrm{mg} \mathrm{dm}^{-3}$ ); 2- Dose $=0$ (solo sem adição de nutrientes); 3 - Ca 
Tabela 1 - Características química e física do solo utilizado na produção das mudas de Dalbergia nigra (Vell.) Allemão ex Benth antes da aplicação dos tratamentos.

Table 1 - Chemical and physical analysis of the soil used in the production of seedlings of Dalbergia nigra (Vell.) Allemão ex Benth before the application of the treatments.

\begin{tabular}{|c|c|c|c|c|c|c|c|c|c|c|c|c|}
\hline \multirow[t]{2}{*}{ Tipo de solo } & \multirow[t]{2}{*}{ Classe textural } & \multirow{2}{*}{$\frac{\mathrm{pH}}{\left(\mathrm{H}_{2} \mathrm{O}\right)}$} & $\mathrm{P}$ & K & $\mathrm{Ca}^{2+}$ & $\mathrm{Mg}^{2+}$ & $\mathrm{Al}^{3+}$ & $\mathrm{H}+\mathrm{Al}$ & SB & $(\mathrm{T})$ & $\mathrm{V}$ & $\mathrm{m}$ \\
\hline & & & \multicolumn{2}{|c|}{$\left(\mathrm{mg} \mathrm{dm}^{-3}\right)$} & \multicolumn{6}{|c|}{$\left(\mathrm{cmol}_{\mathrm{c}} \mathrm{dm}^{-3}\right)$} & \multicolumn{2}{|c|}{$(\%)$} \\
\hline LVAd & Muito argilosa & 5,20 & 0,80 & 49 & 0,30 & 0,00 & 0,00 & 1,82 & 0,43 & 2,25 & 19 & 0 \\
\hline PVAm & Franco-argilosa & 6,00 & 2,10 & 14 & 1,60 & 0,10 & 0,00 & 3,63 & 1,74 & 5,37 & 32 & 0 \\
\hline LVAa & Argilosa & 4,80 & 1,40 & 32 & 0,20 & 0,00 & 0,80 & 3,96 & 0,28 & 4,24 & 7 & 74 \\
\hline
\end{tabular}

Sendo: LVAd: Latossolo Vermelho-Amarelo distrófico; PVAm: Argissolo Vermelho-Amarelo mestrófico; LVAa: Latossolo Vermelho-Amarelo álico; pH (1: 2,5) em água. P e K - extrator Mehlich 1 . $\mathrm{Ca}^{2+}, \mathrm{Mg}^{2+}$ e $\mathrm{Al}^{3+}$ - extrator: $\mathrm{KCl} 1 \mathrm{~mol} \mathrm{~L}{ }^{-1} \mathrm{H}+\mathrm{Al}-$ extrator Acetato de Cálcio $0,5 \mathrm{~mol} \mathrm{~L}^{-1}-\mathrm{pH} 7,0$.

$=0,8 \mathrm{cmol}_{\mathrm{c}} \mathrm{dm}^{-3} ; \mathbf{4}-\mathrm{Ca}=1,2 \mathrm{cmol}_{\mathrm{c}} \mathrm{dm}^{-3} ; 5-\mathrm{Ca}=1,4$ $\mathrm{cmol}_{\mathrm{c}} \mathrm{dm}^{-3} ; \mathbf{6}-\mathrm{Mg}=0,2 \mathrm{cmol}_{\mathrm{c}} \mathrm{dm}^{-3} ; 7-\mathrm{Mg}^{-}=0,6 \mathrm{cmol}_{\mathrm{c}}$ $\mathrm{dm}^{-3} ; \mathbf{8}-\mathrm{Mg}=0,8 \mathrm{cmol}_{\mathrm{c}} \mathrm{dm}^{-3} ; \mathbf{9}-\mathrm{S}=20 \mathrm{mg} \mathrm{dm}^{-3} ; \mathbf{1 0}-$ $\mathrm{S}=60 \mathrm{mg} \mathrm{dm}^{-3}$; e $11-\mathrm{c}=80 \mathrm{mg} \mathrm{dm}^{-3}$.

Porções de $2,1 \mathrm{dm}^{3}$ de cada solo foram colocadas em sacos de polietileno de capacidade aproximada de $5 \mathrm{~kg}$. Posteriormente, os sais $\mathrm{CaCO}_{3}$ e $\mathrm{MgCO}_{3}$ em forma de pó foram adicionados nas quantidades definidas nos tratamentos e homogeneizados. Adicionou-se água até a capacidade de campo, e esse teor de umidade foi mantido por 20 dias com monitoramento diário por pesagens do solo mais água. Logo após esse período, a irrigação foi suspensa até os 30 dias para que o solo secasse, possibilitando a adição dos demais nutrientes.

Os demais nutrientes foram adicionados ao solo seco, na forma de uma solução aquosa, com auxílio de pipetas graduadas em mL. Estas foram previamente preparadas, com dissolução dos sais pesados nas quantidades definidas pelos tratamentos e calculados de acordo com a fonte fornecedora, em água destilada. $\mathrm{O} \mathrm{N}$, o $\mathrm{K}$ e o $\mathrm{S}$ foram diluídos quatro vezes, visto que eles foram aplicados parcelados em quatro vezes ( 0 , 30, 60, 90 dias) após a semeadura. Os sais usados no preparo da solução foram: $\mathrm{NH}_{4} \mathrm{H}_{2} \mathrm{PO}_{4}, \mathrm{NH}_{4} \mathrm{NO}_{3},\left(\mathrm{NH}_{4}\right)_{2} \mathrm{SO}_{4}$, $\mathrm{K}_{2} \mathrm{SO}_{4}$ e KCl.

Adicionou-se também, antes da semeadura, uma solução de micronutrientes nas seguintes doses: $\mathrm{B}$ $=0,81 \mathrm{mg} \mathrm{dm}^{-3}\left(\mathrm{H}_{3} \mathrm{BO}_{3}\right), \mathrm{Cu}=1,33 \mathrm{mg} \mathrm{dm}^{-3}\left(\mathrm{CuSO}_{4} \cdot 5 \mathrm{H}_{2} \mathrm{O}\right)$, $\mathrm{Mo}=0,15 \mathrm{mg} \mathrm{dm}^{-3}$ [(NH4) $\left.)_{6} \mathrm{Mo}_{7} \mathrm{O}_{24} \cdot 4 \mathrm{H}_{2} \mathrm{O}\right], \mathrm{Mn}=3,66$ $\mathrm{mg} \mathrm{dm}{ }^{-3}\left(\mathrm{MnCl}_{2} \cdot \mathrm{H}_{2} \mathrm{O}\right)$ e $\mathrm{Zn}=4,0 \mathrm{mg} \mathrm{dm}^{-3}\left(\mathrm{ZnSO}_{4} \cdot 7 \mathrm{H}_{2} \mathrm{O}\right)$ (ALVAREZ V., 1974).

Após a adubação e devida homogeneização, os solos foram acondicionados em vasos de polipropileno rígido, com dimensões aproximadas de $19 \mathrm{~cm}$ de altura,
14 cm do diâmetro maior e $11 \mathrm{~cm}$ do diâmetro menor e capacidade de $2,1 \mathrm{dm}^{3}$, o qual, juntamente com uma muda, consistiu a parcela experimental.

As sementes de jacarandá-da-bahia (Dalbergia nigra (Vell.) Allemão ex Benth) foram adquiridas no Setor de Silvicultura do Departamento de Engenharia Florestal da Universidade Federal de Viçosa, em Viçosa, MG. Cada vaso recebeu 10 sementes, efetuando-se o primeiro desbaste aos 15 dias após a emergência, deixando-se duas plantas por vaso. Após 30 dias da semeadura, um segundo desbaste foi realizado, deixando-se apenas uma planta por vaso. Durante o período experimental, a umidade do solo foi mantida próxima de $60 \%$ da capacidade de campo, procedendo-se ao monitoramento diário com o uso de balança para esse controle, tendo como base a massa de solo e água por vaso.

Medições de altura e diâmetro do coleto das mudas foram realizadas aos 120 dias após a semeadura. Em seguida, as plantas foram colhidas e subdivididas em raízes e parte aérea, lavadas em água destilada e secas em estufa a $45^{\circ} \mathrm{C}$, com circulação forçada de ar até massa em equilíbrio. Depois de secas, foram pesadas em balança analítica com precisão de 0,01 g para determinação da massa seca da parte aérea (MSPA) e das raízes (MSR), e, pelo somatório destas, calculou-se a massa seca total da planta (MST).

Os dados foram submetidos à análise estatística por meio de contrastes, análises de variância e de regressão com o uso dos programas SISVAR (FERREIRA, 2010) e SAEG (Sistema de Análises Estatística e Genética) (EUCLYDES, 1997), respectivamente.

Testou-se o efeito da adição de macronutrientes em contrastes entre as médias do tratamento 2 (dose $=0$, sem a adição de nutrientes) versus as médias dos demais tratamentos, para cada nutriente: $1=-\mathrm{T} 1+4 \mathrm{~T} 2-\mathrm{T} 3-$ 
T4-T5, para comparar com a adição de Ca; $2=-\mathrm{T} 1+4 \mathrm{~T} 2$ -T6-T7-T8, para comparar a adição de Mg; e $3=-\mathrm{T} 1$ +4T2 -T9-T10-T11, para comparar a adição de S.

Para obtenção das equações, utilizaram-se quatro pontos, sendo três relativos às doses testadas para cada nutriente e um tratamento de referência comum para todos os nutrientes. As curvas de respostas à adição de doses de cada nutriente resultaram de modelos de regressão, nos quais os coeficientes foram testados com base nos valores do quadrado médio do resíduo da ANOVA, sendo analisada a 1\%, 5\% e $10 \%$ de probabilidade.
A partir das equações, no caso de modelos quadráticos e de raiz quadrada, foram determinadas as doses recomendadas de enxofre, cálcio e magnésio, para obtenção de $90 \%$ dos valores máximos estimados para as diversas características estudadas.

\section{RESULTADOS}

As médias de crescimento das mudas de jacarandáda-bahia (Tabela 2) variaram tanto em relação à adição dos nutrientes quanto ao tipo de solo. Em ambos os casos, os valores foram maiores no LVAd, intermediários no PVAm e inferiores nas mudas cultivadas no LVAa.

Tabela 2 - Médias observadas para o crescimento em altura (cm), diâmetro (mm) e massa seca (g) da parte aérea (MSPA), massa seca de raiz (MSR) e massa seca total (MST) em mudas de Dalbergia nigra (Vell.) Allemão ex Benth, sob diferentes doses de enxofre, cálcio e magnésio, aos 120 dias após a semeadura, Rio Pomba, MG, 2006.

Table 2 - Observed averages for height ( $\mathrm{cm}$ ), diameter $(\mathrm{mm})$, and dry mass ( $\mathrm{g}$ ) of the shoots (MSPA), root dry mass (MSR) and total dry mass (MST) of seedlings of Dalbergia nigra (Vell.) Allemão ex Benth under different doses of sulfur, calcium and magnesium, 120 days after sowing, Rio Pomba, MG, 2006.

\begin{tabular}{|c|c|c|c|c|c|c|c|}
\hline Solo & Nutrientes & Doses & Diâmetro (mm) & Altura (cm) & MSPA(g) & MSR (g) & MST (g) \\
\hline \multirow[t]{11}{*}{ LVAd } & Referência & $(*)$ & 1,90 & 19,93 & 0,30 & 0,29 & 0,59 \\
\hline & Zero & Sem adição & 1,69 & 11,18 & 0,09 & 0,13 & 0,22 \\
\hline & $\mathrm{Ca}$ & 0,8 & 2,55 & 31,03 & 0,87 & 0,60 & 1,47 \\
\hline & $\mathrm{Ca}$ & 1,2 & 1,99 & 18,35 & 0,28 & 0,32 & 0,59 \\
\hline & $\mathrm{Ca}$ & 1,4 & 2,72 & 23,55 & 0,57 & 0,52 & 1,09 \\
\hline & Mg & 0,2 & 2,09 & 22,08 & 0,29 & 0,30 & 0,59 \\
\hline & $\mathrm{Mg}$ & 0,6 & 2,22 & 19,77 & 0,33 & 0,25 & 0,58 \\
\hline & $\mathrm{Mg}$ & 0,8 & 2,76 & 34,28 & 0,81 & 0,57 & 1,38 \\
\hline & $\mathrm{S}$ & 20 & 2,50 & 22,00 & 0,53 & 0,38 & 0,91 \\
\hline & $\mathrm{S}$ & 60 & 2,58 & 23,15 & 0,44 & 0,47 & 0,91 \\
\hline & $\mathrm{S}$ & 80 & 2,88 & 27,25 & 0,81 & 0,57 & 1,38 \\
\hline \multirow[t]{11}{*}{ PVAm } & Referência & $(*)$ & 4,40 & 48,30 & 2,14 & 1,41 & 3,55 \\
\hline & Zero & Sem adição & 1,71 & 13,13 & 0,08 & 0,10 & 0,17 \\
\hline & $\mathrm{Ca}$ & 0,8 & 3,94 & 38,68 & 1,45 & 1,05 & 2,49 \\
\hline & $\mathrm{Ca}$ & 1,2 & 3,92 & 36,36 & 1,88 & 1,31 & 3,20 \\
\hline & $\mathrm{Ca}$ & 1,4 & 3,99 & 40,33 & 1,63 & 0,81 & 2,44 \\
\hline & $\mathrm{Mg}$ & 0,2 & 3,32 & 32,88 & 0,98 & 0,72 & 1,70 \\
\hline & $\mathrm{Mg}$ & 0,6 & 3,80 & 37,33 & 1,30 & 0,77 & 2,06 \\
\hline & Mg & 0,8 & 3,32 & 33,58 & 1,11 & 0,70 & 1,81 \\
\hline & $\mathrm{S}$ & 20 & 3,98 & 40,85 & 1,92 & 1,11 & 3,03 \\
\hline & $\mathrm{S}$ & 60 & 4,51 & 53,88 & 2,42 & 1,11 & 3,53 \\
\hline & $\mathrm{S}$ & 80 & 5,16 & 50,00 & 2,68 & 1,78 & 4,47 \\
\hline \multirow[t]{11}{*}{ LVAa } & Referência & $(*)$ & 3,86 & 40,43 & 1,47 & 1,25 & 2,72 \\
\hline & Zero & Sem adição & 1,70 & 13,10 & 0,12 & 0,14 & 0,25 \\
\hline & $\mathrm{Ca}$ & 0,8 & 5,08 & 63,55 & 3,36 & 2,12 & 5,47 \\
\hline & $\mathrm{Ca}$ & 1,2 & 5,72 & 75,18 & 3,96 & 1,88 & 5,83 \\
\hline & $\mathrm{Ca}$ & 1,4 & 5,04 & 55,13 & 3,05 & 1,71 & 4,77 \\
\hline & $\mathrm{Mg}$ & 0,2 & 5,63 & 57,65 & 3,37 & 1,91 & 5,28 \\
\hline & Mg & 0,6 & 4,67 & 56,63 & 1,53 & 1,51 & 3,04 \\
\hline & Mg & 0,8 & 5,82 & 66,45 & 3,95 & 2,16 & 6,11 \\
\hline & $\mathrm{S}$ & 20 & 5,36 & 67,90 & 3,39 & 2,02 & 5,41 \\
\hline & $\mathrm{S}$ & 60 & 5,36 & 51,33 & 2,82 & 1,40 & 4,22 \\
\hline & $\mathrm{S}$ & 80 & 5,16 & 54,18 & 2,89 & 1,64 & 4,53 \\
\hline
\end{tabular}

$\left(^{*}\right) \mathrm{N}=100 \mathrm{mg} \mathrm{dm}^{-3}, \mathrm{P}=300 \mathrm{mg} \mathrm{dm}^{-3}, \mathrm{~K}=100 \mathrm{mg} \mathrm{dm}^{-3}, \mathrm{Ca}=1 \mathrm{cmol}_{\mathrm{c}} \mathrm{dm}^{-3}, \mathrm{Mg}=0,4 \mathrm{cmol}_{\mathrm{c}} \mathrm{dm}^{-3}, \mathrm{~S}=40 \mathrm{mg} \mathrm{dm}^{-3}$

Revista Árvore, Viçosa-MG, v.38, n.2, p.251-260, 2014 
Pelas estimativas dos contrastes (Tabela 3), só foi benéfica a aplicação de cálcio, quando se avaliaram as características altura, massa seca de raiz e massa seca total, quando as mudas foram cultivadas no solo LVAd, não sendo observado efeito da aplicação de Mg e S em nenhuma das características quando as mudas foram cultivadas nesse solo. No PVAm foram observados efeitos em grande parte das características estudadas, estando ausentes apenas na massa seca da parte área, para os três nutrientes, e na massa seca total quando se aplicou Mg. No entanto, as mudas foram todas beneficiadas pela aplicação de $\mathrm{Ca}, \mathrm{Mg}$ e S quando cultivadas no LVAa.

Verificaram-se efeitos significativos da adição de cálcio no LVAd sobre as características diâmetro, massa seca de raiz e massa seca total (Tabela 4). Porém, a resposta da planta obtida no experimento não possibilitou ajustar modelos que permitissem definir as doses que promoveriam $90 \%$ do crescimento máximo. No PVAm só se observaram influência no crescimento das mudas de jacarandá-da-bahia, na massa seca da raiz, cujo comportamento quadrático verificado permitiu estimar a dose para garantir $90 \%$ da produção em $0,9 \mathrm{cmol}_{\mathrm{c}} \mathrm{dm}^{-3}$. As diferentes doses de cálcio aplicadas ao solo LVAa não implicaram efeitos significativos (Tabela 4).

A adição de magnésio no solo foi significativa com efeito linear nas características diâmetro, massa seca da parte aérea e massa seca total quando as plantas foram cultivadas no LVAd, não se verificando efeito no crescimento de raízes, sendo a média observada dessa característica de 0,35 g quando cultivadas nesse solo.

O efeito observado nas mudas plantadas no PVAm, em razão de crescentes doses de magnésio, foi significativo para diâmetro e altura, com modelos ajustados de raiz quadrada. Para atingir 90\% da produção máxima do diâmetro $(3,80 \mathrm{~mm})$, a dose recomendada desse nutriente é de $0,26 \mathrm{cmol}_{\mathrm{c}} \mathrm{dm}^{-3}$, e para altura, de $0,27 \mathrm{cmol}_{\mathrm{c}} \mathrm{dm}^{-3}$ para se obterem 39,8 $\mathrm{cm}$. As características massa seca de raiz, parte aérea e total não foram influenciadas.

O comportamento do crescimento das mudas plantadas no LVAa foi caracterizado por modelos ajustados de raiz quadrada para o diâmetro, altura e massa seca de raiz e quadrático para a massa seca da parte aérea e total. Entretanto, não foi possível explicar a curva de crescimento, por esta não apresentar realismo biológico, não sendo possível determinar uma dose adequada.

Pela Tabela 4, observa-se que no LVAd não foram observados efeitos significativos da adição das diferentes doses de enxofre. Contudo, a aplicação de enxofre provocou efeitos significativos no crescimento das mudas plantadas no PVAm, nas características diâmetro do colo e massa seca de raiz, sendo o modelo linear o que melhor explicou tal efeito. No LVAa, não foram encontrados modelos matemáticos que se ajustassem adequadamente às respostas observadas e significativas para as características de crescimento, à exceção do diâmetro que não foi significativo.

Tabela 3 - Estimativas dos contrastes entre o tratamento sem adição de sais e os demais tratamentos, em que se adicionaram macronutrientes.

Table 3 - Estimates of the contrasts between the treatment without salts and the other treatments, with the addition of macronutrients.

\begin{tabular}{|c|c|c|c|c|c|c|}
\hline \multirow[b]{2}{*}{ Nutriente } & \multirow[b]{2}{*}{ SOLO } & \multicolumn{4}{|c|}{ Estimativas do contraste } & \multirow[b]{2}{*}{ MST(g) } \\
\hline & & Diâmetro (mm) & Altura (cm) & MSPA(g) & MSR(g) & \\
\hline \multirow{3}{*}{$\mathrm{Ca}$} & LVAd & $-0,48^{n s}$ & $-10,79^{*}$ & $-0,40^{\mathrm{ns}}$ & $-0,28 *$ & $-0,68^{*}$ \\
\hline & PVAm & $-2,08^{* * *}$ & $-24,77^{* * *}$ & $-2,69 \mathrm{~ns}$ & $-0,96^{* * *}$ & $-3,65^{*}$ \\
\hline & LVAa & $-3,22^{* * *}$ & $-45,47^{* * *}$ & $-2,84^{* * *}$ & $-1,60^{* * *}$ & $-4,44^{* * *}$ \\
\hline \multirow{3}{*}{$\mathrm{Mg}$} & LVAd & $-0,29 \mathrm{~ns}$ & $-10,36^{*}$ & $-0,31^{\mathrm{ns}}$ & $-0,19 \mathrm{~ns}$ & $-0,50 \mathrm{~ns}$ \\
\hline & PVAm & $-1,73^{* * *}$ & $-21,86^{* * *}$ & $-1,17$ ns & $-0,71^{* *}$ & $-1,86$ ns \\
\hline & LVAa & $-3,29^{* * *}$ & $-42,19^{* * *}$ & $-2,47^{* * *}$ & $-1,57^{* * *}$ & $-4,03^{* * *}$ \\
\hline \multirow{3}{*}{$\mathrm{S}$} & LVAd & $-0,17^{\mathrm{ns}}$ & $-6,39 \mathrm{~ns}$ & $-0,33^{n s}$ & $-0,19$ ns & $-0,52 \mathrm{~ns}$ \\
\hline & PVAm & $-2,53^{* * *}$ & $-32,11^{* * *}$ & $-2,08^{n s}$ & $-1,17^{* * *}$ & $-3,25 *$ \\
\hline & LVAa & $-3,23^{* * *}$ & $-40,36^{* * *}$ & $-2,53^{* * *}$ & $-1,44^{* * *}$ & $-3,96^{* * *}$ \\
\hline
\end{tabular}

*** significativo a $1 \%$ de probabilidade, ** significativo a $5 \%$ de probabilidade * ${ }^{*}$ ignificativo a $10 \%$ de propabilidade e ${ }^{\text {ns }}$ não significativo. 
Tabela 4 - Equações de regressão e doses estimadas de enxofre, cálcio e magnésios para altura, diâmetro do colo, massa seca da raiz, partes aérea e total na produção de mudas de Dalbergia nigra (Vell.) Allemão ex Benth aos 120 dias após a semeadura, Rio Pomba, MG, 2006.

Table 4 - Regression equations and estimated doses of sulfur, calcium and Magnesium for height, stem diameter, root dry mass, shoots and total production of seedlings of Dalbergia nigra (Vell.) Allemão ex Benth at 120 days after sowing, Rio Pomba, MG, 2006.

\begin{tabular}{|c|c|c|c|c|}
\hline$\underline{\text { Solo }}$ & Característica & Equação & $\mathrm{R}^{2}$ & Dose Recomendada \\
\hline & & Cálcio & & $\left(\mathrm{cmol}_{\mathrm{e}} \mathrm{dm}^{-3}\right)$ \\
\hline \multirow[t]{5}{*}{ LVAd } & Diâmetro (mm) & $\begin{array}{l}\hat{Y}=11,9119-18,5835 * * X+ \\
8,58655 * * X^{2}\end{array}$ & 0,99 & - \\
\hline & Altura (cm) & $\hat{Y}=Y=23,21$ & - & 0,80 \\
\hline & MSPA (g) & $\hat{Y}=\mathrm{Y}=0,50$ & - & 0,80 \\
\hline & MSR (g) & $\begin{array}{l}\hat{Y}=4,24-7,09987 * * X+ \\
3,17682 * * X^{2}\end{array}$ & 0,98 & - \\
\hline & MST (g) & $\begin{array}{l}\hat{Y}=11,4202-19,2521 * * X+ \\
8,48918 * * X^{2}\end{array}$ & 0,31 & - \\
\hline \multirow[t]{5}{*}{ PVAm } & Diâmetro (mm) & $\hat{Y}=\mathrm{Y}=4,06$ & - & 0,80 \\
\hline & Altura $(\mathrm{cm})$ & $\hat{\mathrm{Y}}=\mathrm{Y}=40,91$ & - & 0,80 \\
\hline & MSPA (g) & $\hat{Y}=\mathrm{Y}=1,77$ & - & 0,80 \\
\hline & $\operatorname{MSR}(\mathrm{g})$ & $\begin{array}{l}\hat{Y}=-4,72188+11,5649 * * X- \\
5,44098 * * X^{2}\end{array}$ & 0,99 & 0,90 \\
\hline & $\operatorname{MST}(\mathrm{g})$ & $\vec{Y}=\mathrm{Y}=2,92$ & - & 0,80 \\
\hline \multirow[t]{6}{*}{ LVAa } & Diâmetro (mm) & $\hat{Y}=Y=5,28$ & - & 0,80 \\
\hline & Altura (cm) & $\hat{Y}=\mathrm{Y}=58,57$ & - & 0,80 \\
\hline & MSPA (g) & $\hat{Y}=\mathrm{Y}=3,45$ & - & 0,80 \\
\hline & MSR (g) & $\hat{Y}=\mathrm{Y}=1,90$ & - & 0,80 \\
\hline & $\operatorname{MST}(\mathrm{g})$ & $\hat{Y}=Y=5,36$ & - & 0,80 \\
\hline & & Magnésio & & $\left(\mathrm{cmol}_{c} \mathrm{dm}^{-3}\right)$ \\
\hline \multirow[t]{5}{*}{ LVAd } & Diâmetro (mm) & $\hat{Y}=1,6533+1,1742 * X$ & 0,67 & 0,80 \\
\hline & Altura $(\mathrm{cm})$ & $\begin{array}{l}\hat{Y}=90,2217-239,136 * * X^{0,5}+ \\
196,309 * * X\end{array}$ & 0,34 & 0,20 \\
\hline & MSPA (g) & $\hat{Y}=0,0342+0,7971 * * X$ & 0,67 & 0,80 \\
\hline & $\operatorname{MSR}(\mathrm{g})$ & $\hat{Y}=\mathrm{Y}=0,35$ & - & 0,20 \\
\hline & $\operatorname{MST}(\mathrm{g})$ & $\hat{Y}=0,1929+1,1838 * X$ & 0,60 & 0,80 \\
\hline \multirow[t]{5}{*}{ PVAm } & Diâmetro $(\mathrm{mm})$ & $\begin{array}{l}\hat{Y}=-3,85939+24,3435 * X^{0,5}- \\
18,343 * X\end{array}$ & 0,28 & 0,26 \\
\hline & Altura $(\mathrm{cm})$ & $\begin{array}{l}\hat{Y}=-55,2067+300,114 * X^{0,5}- \\
226,550 * X\end{array}$ & 0,30 & 0,27 \\
\hline & MSPA (g) & $\hat{Y}=\mathrm{Y}=1,38$ & & 0,20 \\
\hline & $\operatorname{MSR}(\mathrm{g})$ & $\hat{Y}=\mathrm{Y}=0,90$ & - & 0,20 \\
\hline & $\operatorname{MST}(\mathrm{g})$ & $\hat{Y}=\mathrm{Y}=2,28$ & & 0,20 \\
\hline \multirow[t]{6}{*}{ LVAa } & Diâmetro (mm) & $\begin{array}{l}\hat{Y}=19,1625-45,8729 * X^{0,5}+ \\
34,7077 * X\end{array}$ & 0,33 & - \\
\hline & Altura $(\mathrm{cm})$ & $\begin{array}{l}\hat{Y}=184,799-443,230 * X^{0,5}+ \\
350,128 * X\end{array}$ & 0,22 & - \\
\hline & MSPA (g) & $\begin{array}{l}\hat{Y}=7,53313-26,1144 * * * X+ \\
27,0156 * * * X^{2}\end{array}$ & 0,99 & - \\
\hline & MSR (g) & $\begin{array}{l}\hat{Y}=7,82296-20,1531 * * X^{0,5}+ \\
15,4639 * * X\end{array}$ & 0,29 & - \\
\hline & $\operatorname{MST}(\mathrm{g})$ & $\begin{array}{l}\hat{Y}=10,6206-33,7894 * * * X+ \\
35,2031 * * * X^{2}\end{array}$ & 0,48 & - \\
\hline & & Enxofre & & $\left(\mathrm{mg} \mathrm{dm}^{-3}\right)$ \\
\hline \multirow[t]{2}{*}{ LVAd } & Diâmetro (mm) & $\hat{Y}=\mathrm{Y}=2,46$ & - & 20 \\
\hline & Altura $(\mathrm{cm})$ & $\hat{Y}=Y=23,08$ & - & 20 \\
\hline
\end{tabular}

Revista Árvore, Viçosa-MG, v.38, n.2, p.251-260, 2014 
Tabela 4 - Cont.

Table 4-Cont

\begin{tabular}{|c|c|c|c|c|}
\hline & MSPA (g) & $\hat{Y}=Y=0,52$ & - & 20 \\
\hline & MSR (g) & $\hat{Y}=\mathrm{Y}=0,43$ & - & 20 \\
\hline & MST (g) & $\hat{Y}=\mathrm{Y}=0,95$ & - & 20 \\
\hline PVAm & Diâmetro (mm) & $\hat{Y}=3,6017+0,0182 * X$ & 0,93 & 80 \\
\hline & Altura $(\mathrm{cm})$ & $Y=Y=48,270$ & - & 20 \\
\hline & MSPA (g) & $Y=Y=2,29$ & - & 20 \\
\hline & MSR (g) & $\hat{Y}=0,9204+0,0087 * X$ & 0,49 & 80 \\
\hline & $\operatorname{MST}(\mathrm{g})$ & $Y=Y=3,64$ & - & 20 \\
\hline LVAa & Diâmetro $(\mathrm{mm})$ & $\hat{Y}=Y=5,28$ & - & 20 \\
\hline & Altura $(\mathrm{cm})$ & - & - & - \\
\hline & MSPA (g) & - & - & - \\
\hline & MSR (g) & - & - & - \\
\hline & $\operatorname{MST}(\mathrm{g})$ & - & - & - \\
\hline
\end{tabular}

\section{DISCUSSÃO}

\subsection{Efeito do cálcio}

Os teores desse nutriente encontrado originalmente no solo LVAd eram muito baixos, conforme Alvarez V. et al. (1999), mas podem ter suprido as necessidades das mudas, uma vez que as curvas de crescimento obtidas, para as características diâmetro do colo, massa seca de raiz e massa seca total, não explicaram o efeito, e as demais características não foram influenciadas, Nesse solo, verificou-se, pela análise das estimativas dos contrastes, que não fez diferença aplicar ou não o cálcio para as características diâmetro e massa seca da parte aérea. Isso corrobora com Raij (1991), que afirmou que, em grande parte das culturas, o efeito da aplicação do cálcio não tem sido registrado com frequência, em parte pelo fato de as espécies não serem tão exigentes desse nutriente.

No solo PVAm, a despeito de os teores de Ca originalmente encontrados serem considerados médios, o contraste com as mudas que receberam adição de cálcio indicou ser benéfica a aplicação desse nutriente nesse solo. Entretanto, somente se observaram efeito na massa seca das raízes das mudas. Tal fato indica que deve ser aplicado Ca nesse solo, pelo menos na quantidade mínina testada, que é de $0,80 \mathrm{cmol}_{\mathrm{c}} \mathrm{dm}^{-3}$ até se realizaram novos testes com doses menores.

Da mesma forma, no LVAa os teores originalmente presentes nesse solo eram muito baixos, mas, ainda assim, não se verificou efeito da adição do cálcio, ao se compararem as doses aplicadas. Todavia, pela interpretação do contraste, verificou-se ser importante a aplicação do cálcio para o crescimento das mudas, visto que estas cresceram mais nos tratamentos em que se adicionou o nutriente em relação ao tratamento sem adição de qualquer nutriente. Logo, optou-se por recomendar a menor dose testada até que novos estudos, com doses inferiores a essa, fossem realizados.

Os resultados dos três solos em questão sinalizaram que a necessidade de Ca pela espécie é baixa, em comparação com tabelas dos manuais de recomendação. É possível que a dose ideal seja um valor abaixo da menor dose testada. Essas baixas quantidades requeridas de Ca ou, mesmo, a não necessidade da aplicação desse nutriente, podem estar relacionadas, também, ao fato de o jacarandá-da-bahia ser uma espécie de classificação sucessional secundária tardia a clímax. Tal comportamento, segundo Gonçalves et al. (1992), sugeriu menor demanda nutricional na fase inicial de crescimento. Uma comprovação desse fato foi observada em mudas de jatobá (Hymenaea courbaril), em que a omissão de cálcio não afetou o crescimento das mudas de maneira significativa, tendo o crescimento no tratamento com a omissão do nutriente sido muito semelhante ao do tratamento completo (DUBOC et al., 1996).

Baixa resposta à aplicação de Ca também foi observada em mudas de angico-vermelho (Anadenanthera macrocarpa) cultivadas em Argissolo Vermelho-Amarelo, Latossolo Vermelho-Amarelo álico e Latossolo VermelhoAmarelo distrófico (GONÇALVES et al., 2008).

Todavia, diferentemente deste trabalho, outros autores como Santanna et al. (1996), em um Latossolo Vermelho-Amarelo distrófico, mostraram efeito significativo da adição de cálcio ao substrato em todos

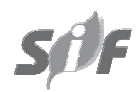

Revista Árvore, Viçosa-MG, v.38, n.2, p.251-260, 2014 
os parâmetros avaliados no trabalho com seringueira. Similarmente, mudas de angico-amarelo (Peltophorum dubium) cultivadas em Latossolo Vermelho-Amarelo tiveram o seu crescimento em altura e diâmetro afetado pela omissão de cálcio no meio de crescimento, tendo ainda a produção de massa seca tanto da parte aérea quanto da raiz, tendo sido menores quando se omitiu o nutriente (VENTURIN et al., 1999).

\subsection{Efeito do magnésio}

O comportamento da muda sob influência da aplicação de Mg no LVAa variou em razão das diferentes doses aplicadas, e o nutriente só não afetou a característica massa seca da raiz. As demais foram afetadas de forma linear, com maior crescimento na maior dose testada. Entretanto, embora tenha sido observada diferença de crescimento entre as doses, em média, esse crescimento não foi maior do que o ocorrido no tratamento sem adição de nutrientes, pois, pela análise do contraste, a exceção da característica altura, não fez diferença aplicar ou não o nutriente. Vale ressaltar que, inicialmente, esse solo já apresentava a melhor fertilidade natural entre os demais. Isso sugere que pode ter ocorrido consumo de luxo pela planta e que, na verdade, ela não necessita de grandes doses de $\mathrm{Mg}$.

No PVAm, os teores de Mg encontrados originalmente eram muito baixos (ALVAREZ V., 1999), e só o diâmetro e a altura foram influenciados. A quantidade recomendada de 0,26 e 0,27 $\mathrm{mg} \mathrm{dm}^{-3}$, obtida após a interpretação da curva, ainda é um teor baixo segundo os manuais de recomendação de adubação. Essa resposta sugere que a espécie seja pouco exigente desse nutriente, mas é importante a aplicação deste em solos com características parecidas, pois as mudas cresceram mais com o adição de $\mathrm{Mg}$ ao solo, em relação às que cresceram no solo puro, tal como foi verificado pelo contraste (Tabela 3).

No LVAa, pela estimativa do contraste, verificou que as mudas cresceram mais nos tratamentos com adição de $\mathrm{Mg}$, o que era esperado, em razão de o teor inicial ser zero. Contudo, os efeitos representados pelos modelos ajustados não apresentaram coerência biológica. Portanto, não foi possível definir a melhor dose.

Na literatura há poucos estudos avaliando separadamente o efeito do magnésio no crescimento das mudas, pois geralmente enfocam a relação Ca:Mg, gerando resultados para essa relação e não para cada nutriente em separado, o que dificulta a comparação com os resultados deste estudo. Entretanto, é importante a nutrição das plantas com esse nutriente, pois este tem, entre as suas várias funções, a de ativador enzimático nas reações de transferência de fosfato ou nucleotídios, de grupos carboxílicos e de ativadores de desidrogenase, mutase e litase. Também, tem papel estrutural como componente da molécula de clorofila (DECHEN; NACHTIGALL, 2007).

Respostas diferenciadas à aplicação de magnésio têm sido, todavia, observadas tal como em resultados de Renó et al. (1997), que verificaram em quatro espécies florestais respostas diferenciadas à omissão de magnésio quando cultivadas em um Latossolo Vermelho-Amarelo, sendo a omissão do nutriente restritiva ao crescimento do pau-ferro (Caesalpinea ferrea) e do cedro (Cedrela fissilis), não tendo afetado o crescimento do jacaré (Piptadenia gonoacantha) e da canafístula (Senna multijuga).

Da mesma forma que para essas duas últimas espécies, mudas de óleo-de-copaíba (Copaifera langsdorffii) cultivadas também em Latossolo Vermelho-Amarelo mostraram um pequeno requerimento nutricional para magnésio, não tendo o crescimento em altura e diâmetro das plantas sob a omissão do nutriente diferido daquelas submetidas ao tratamento completo (DUBOC et al., 1996). No entanto, para a produção de massa seca de parte aérea, as mudas de óleo-de-copaíba produzidas no tratamento com omissão de magnésio foram menores quando comparadas com as do tratamento completo.

\subsection{Efeito do enxofre}

O estudo não conseguiu explicar, de forma satisfatória, o efeito do S nos três solos. É possível que os teores originais desse nutriente, embora desconhecido, tenham suprido a necessidade das mudas cultivadas no LVAd, uma vez que não foram observados efeitos da aplicação desse nutriente nem pelo contraste. No PVAm e no LVAa, cujos solos apresentaram pior fertilidade natural, a aplicação do $\mathrm{S}$ foi benéfica, pois as mudas cresceram mais nessa condição do que no solo sem a adição do nutriente. Entretanto, para o PVAm, as curvas indicaram efeito apenas no diâmetro e a massa seca de raiz, nas maiores doses. Nas mudas que cresceram no LVAa, não foi possível ajustar modelos que explicassem os efeitos observados. 
A indicação de doses acima de $80 \mathrm{mg} \mathrm{dm}^{-3}$, observada nas características diâmetro e massa seca da raiz, tal como foi observado nas mudas cultivas no PVAm, também foi verificada em trabalho de Cruz et al. (2010) com mudas de fedegoso (Senna macranthera) cultivadas em um Latossolo Vermelho-Amarelo distrófico. Essas doses são maiores do que as encontradas de $A$. holosericea, cultivadas em Latossolo Vermelho-Amarelo álico, em que a dose recomendável de enxofre no que se refere à produção de massa seca de parte aérea foi de $20 \mathrm{mg} \mathrm{dm}^{-3}$ (BALIEIRO et al., 2001).

Respostas de melhor crescimento de espécies vegetais em razão do suprimento do enxofre têm sido frequentes em experimentos realizados no Brasil e em outras regiões tropicais (ALVAREZ V., 1974). Adicionalmente, outros autores, como Braga et al. (1995), em um solo com as características semelhantes às deste estudo, verificaram que a omissão do enxofre afetou o crescimento das espécies A. mangium, quaresmeira (Tibouchina granulosa); peroba-rosa (Aspidosperma polyneuron); e plantas de pereira (Platycyamus regnellii). Da mesma forma, o nutriente foi altamente limitante para o crescimento de mudas de cedro (Cedrela fissilis), jacaré (Piptadenia gonoacantha), pau-ferro (Caesalpinea férrea) e canafístula (Senna multijuga) em um Latossolo Vermelho-Amarelo de fertilidade natural muito baixa, conforme foi verificado por Renó et al. (1997). A altura e o diâmetro de mudas de angico-amarelo (Peltophorum dubium) também foram afetados pela omissão de enxofre, tendo esses parâmetros alcançados maiores valores no tratamento completo quando comparado ao tratamento da omissão do nutriente, tendo dessa forma afetado o crescimento das mudas (VENTURIN et al., 1999).

Reis et al. (1997), entretanto, encontraram respostas contrárias à desses autores, indicando que o jacarandáda-bahia respondeu, de forma negativa, à aplicação de doses de enxofre ao solo. Da mesma forma, a falta de respostas à aplicação de diferentes doses de enxofre, nas diversas características de crescimento usados na avaliação, também já foi verificada em mudas de táxi-branco (Sclerolobium paniculatum) cultivadas em um Latossolo Vermelho-Amarelo (DIAS et al., 1992) e em mudas de angico-vermelho (Anadenanthera macrocarpa) cultivadas no mesmo solo deste estudo (GONÇALVES et al., 2008). Nesse caso, também se indicou a dose de $20 \mathrm{mg} \mathrm{dm}^{-3}$ para a produção das mudas, visto que a sua não aplicação implicou menores médias de crescimento.

\section{CONCLUSÕES}

O jacarandá-da-bahia se mostrou, em geral, pouco exigente em relação à aplicação dos nutrientes $\mathrm{Ca}, \mathrm{Mg}$ e S. Sendo necessários novos estudos, com doses inferiores às doses testadas nesse estudo, para melhor elucidar a dose requerida.

Enquanto esses estudos não são feitos, para a produção de mudas dessa espécie com qualidade, em condições similares à desse experimento, e tomando como base a massa seca total, indicam-se as doses

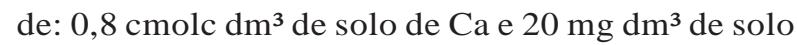
de $\mathrm{S}$, independente do solo testado; e 0,2 e 0,8 cmolc $\mathrm{dm}^{3}$ de solo de Mg para solos com características similares ao PVAm e LVAd, respectivamente.

\section{REFERÊNCIAS}

ALVARES V., V. H. et al.. Interpretação dos resultados das análises de solo. In: RIBEIRO, A.C.; GUIMARAES, P. T. G.; ALVAREZ V., V. H. (Ed.). Recomendação para o uso de corretivos e fertilizantes em Minas Gerais: 5. Aproximação. Viçosa, MG: Comissão de Fertilidade do Solo do Estado de Minas Gerais, 1999. p.25-32.

\section{ALVAREZ V., H. V. Equilíbrio de formas disponíveis de fósforo e enxofre em dois Latossolos de Minas Gerais. 1974. 125f. Dissertação (Mestrado em Solos e Nutrição de Plantas) - Universidade Federal de Viçosa, Viçosa, MG, 1974.}

BALIERO, F. C.; OLIVEIRA, I. G.; DIAS, L. E. Formação de mudas de Acacia holosericea e Acacia auriculiformis : resposta a calagem, fósforo, potássio e enxofre. Revista Árvore, v.25, n.2, p.183-191, 2001.

BERNARDINO, D. C. S. et al. Influência da saturação por bases e da relação Ca:Mg do substrato sobre o crescimento inicial de jacarandá-da-bahia (Dalbergia nigra (Vell.) Fr. All. Ex Benth). Revista Árvore, v.31, n.4, p.567573, 2007.

BRAGA, F. A. et al. Exigências nutricionais de quatro espécies florestais. Revista Árvore, v.19, n.1, p.18-31, 1995.

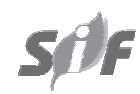

Revista Árvore, Viçosa-MG, v.38, n.2, p.251-260, 2014 
CARVALHO, P. E. R. Espécies arbóreas brasileiras. Brasília: Embrapa Informação Tecnológica/Colombo: Embrapa Florestas, 2003. v.1. 1039p.

CRUZ, C. A. F. et al. Resposta de mudas de Senna macranthera (Dc. Ex Collad.) H.S. Irwin \& Barnaby (Fedegoso) cultivadas em Latossolo Vermelho-Amarelo distrófico a macronutrientes. Rervista Árvore, v.34, n.1, p.13-24, 2010.

DECHEN, A. R.; NACHTIGALL, G. R. Elementos requeridos à nutrição de plantas. In: NOVAIS, R. F. et al. (Ed). Fertilidade do solo. Viçosa, MG: Sociedade Brasileira de Ciência do Solo, 2007. p.91-32.

DIAS, L. E. et al. Formação de mudas de táxi-branco (Sclerolobium paniculatum Voguel): Resposta a nitrogênio, potássio e enxofre. Revista Árvore, v.16, n.2, p.135-143, 1992.

DUBOC, E. et al. Nutrição do jatobá (Hymenaea coubaril L. var. stilbocarpa (Hayne)Lee et Lang).Cerne, v.2, n.1, p.31-47, 1996.

EUCLYDES, R. F. Manual de utilização do programa SAEG. Viçosa, MG: Universidade Federal de Viçosa, 1997. 59p.

FERREIRA, D. F. Programa computacional SISVAR. versão5.3. Lavras: Universidade Federal de Lavras, 2010.

GONÇALVES, E. O. et al. Crescimento de mudas de angico-vermelho (Anadenanthera macrocarpa (Benth.) Brenan) sob diferentes doses de macronutrientes. Revista Árvore, v.32, n.6, p.1029-1040, 2008.

LORENZI, H. Árvores brasileiras. Nova Odessa: Plantarum, 2000. v.1. 352p
MARQUES, V. B. et al. Efeito de fontes e doses de nitrogênio sobre o crescimento inicial e qualidade de mudas de jacarandá-da-bahia (Dalbergia nigra (Vell.) Fr. All. ex Benth.). Revista Árvore, v.30, n.5, p.725-735, 2006.

RAIJ, B. van. Fertilidade do solo e adubação. Piracicaba: Ceres, Potafos, 1991. 343p.

REIS, M. G. F. et al. Exigências nutricionais de mudas de Dalbergia nigra (Vell.) Fr. Allem (Jacarandá-da-Bahia) produzidas em dois níveis de sombreamento. Revista Árvore, v.21, n.4, p.463-471, 1997.

RENÓ, N. B. et al. Limitações nutricionais ao crescimento inicial de quatro espécies arbóreas nativas em Latossolo Vermelho-Amarelo.

Pesquisa Agropecuária Brasileira, v.32, n.1, p.17-25, 1997.

RESENDE, M. et al. Pedologia: base para distinções de ambientes. 4.ed. Viçosa, MG: Neput, 2002. 338 p.

SANTANNA, C. A. F. et al. Produção de matéria seca em mudas de seringueira submetidas a diferentes doses de fósforo. In: REUNIÃO BRASILEIRA DE FERTILIDADE DO SOLO E NUTRIÇÃO DE PLANTAS, 22., 1996, Manaus. Resumos expandidos... Manaus: Universidade de Manaus, 1996. p.337-338.

TURRENT, F.A. Uso de uma matriz mixta para la optimización de cinco a ocho factores controlables de la producción. Chapingo-México: Rama de Suelos, Colégio de Postgraduados, 1979. 65p. (Boletim Técnico, 6)

VENTURIN, N. et al. Adubação mineral do Angico-Amarelo (Peltophorum dubium (SPRENG.) TAUB.). Pesquisa Agropecuária Brasileira, v.34, n.3, p.441-448, 1999. 